\title{
Deformable Porous Media with Double Porosity. Quasi-Statics. I: Coupling Effects
}

\author{
J. L. AURIAULT \\ Institut de Méchanique de Grenoble, UMR 101, Université Joseph Fourier, \\ Institut National Polytechnique de Grenoble, CNRS, BP 53 X, \\ F-3804I Grenoble Cedex, France \\ and \\ C. BOUTIN \\ Ecole National des Travaux Publics de l'Etat, Labortoire Géometériaux, rue M. Audin, \\ F-69518 Vaulx en Velin Cedex, France
}

\begin{abstract}
We investigate the macroscopic quasi-static description of a deformable porous medium with a double porosity constituted by pores and fractures. For this purpose, we use an homogenization technique which gives the macroscopic modelling from the description at the pore and fracture levels. It appears that the macroscopic description is sensitive to the ratios between the different scales, $l / l^{\prime}$ and $I^{\prime} / l^{\prime \prime}$, where $l, l^{\prime}, l^{\prime \prime}$ are characteristic lengths of the pores, the fractures and the macroscopic medium, respectively. In the first paper we investigate the case $l^{\prime} / l^{\prime \prime}=\left(l / l^{\prime}\right)^{2}$, which exhibits a coupling between the flows through the pores and the fractures. The macroscopic description is shown to depend on a single pressure field and exhibits a broken symmetry. Other situations will be examined in a subsequent paper. Large spectra of pore and fracture sizes are also evoked.
\end{abstract}

Key words. Porous media, double porosity, consolidation.

\section{Nomenclature}

Roman Letters

a

$c^{\prime}, c^{\prime \prime}$

D

e

$f$

$\mathbf{k}_{p}, \mathbf{k}_{f}$

$\mathbf{K}_{p}, \mathbf{K}_{f}$

I

$l, l^{\prime}, l^{\prime \prime}$

$n, n^{\prime}$

$\mathbf{N}$ elastic tensor of the skeleton material, effective elastic tensors of the skeleton, rate of deformation tensor, deformation tensor, subscript for the fractures, particular solutions for the velocity field $\mathbf{v}$ in the pores and the fractures, respectively, filtration tensors of the pores and the fractures, respectively, unit tensor, characteristic lengths of the pore scale, the fracture scale and the macroscopic medium, respectively, pore porosity and fracture porosity, respectively, normal unit vector, 


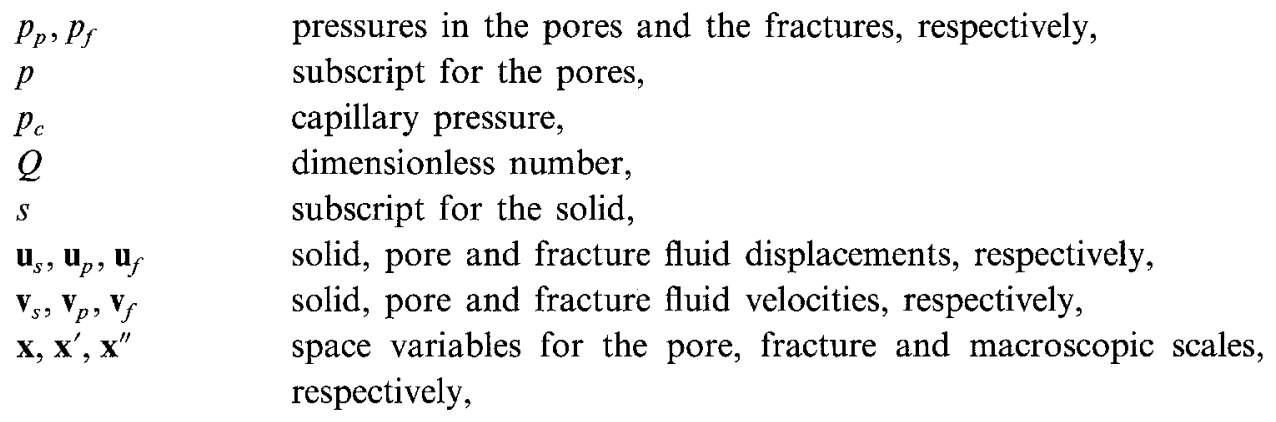

Greek Letters

$\alpha^{\prime}, \alpha^{\prime \prime}, a^{*}, \gamma^{\prime}, \gamma^{\prime \prime}$ coupling effective tensors entering the behavior relations of the porous medium,
$\beta^{\prime}, \beta^{\prime \prime} \quad$ coupling effective scalars entering the behavior relations of the porous medium,
$\Gamma, \Gamma^{\prime} \quad$ boundaries of the pores and the fractures, respectively,
$\Delta \quad$ Laplace operator,
$\nabla \quad$ gradient operator,
$\varepsilon \quad$ small parameter of the homogenization process,
$\eta, \eta^{\prime} \quad$ particular solutions for the displacement field $\mathbf{u}$,
$v^{\prime} \quad$ particular solutions for the displacement field $\mathbf{u}$,
$\mu \quad$ viscosity,
$\xi, \xi^{\prime}, \xi^{*} \quad$ particular solutions for the displacement field $\mathbf{u}$,
$\sigma_{s}, \sigma_{p}, \sigma_{f} \quad$ solid, pore and fracture fluid stress tensors, respectively,
$\tau$ particular solutions for the pressure,
$\Omega, \Omega^{\prime} \quad$ periods at the pore and fracture scales, respectively,
$\Omega_{s}, \Omega_{p}, \Omega_{s p}^{\prime}, \Omega_{f}^{\prime} \quad$ parts of the periods occupied by the solid, the pores, the solid plus the pores and the fractures respectively.

Particular Symbols

$\langle\Phi\rangle_{\Omega},\langle\Phi\rangle_{\Omega^{\prime}},\left\langle\langle\Phi\rangle_{\Omega \Omega^{\prime}}\right.$

volume averages of the quantity $\Phi$ on $\Omega, \Omega^{\prime}$, and $\Omega$ plus $\Omega^{\prime}$, respectively.

\section{Introduction}

We consider a deformable porous medium made up of two porosity systems undergoing a quasi-static excitation. One of the porosity systems is concerned with the pores between the grains of the matrix and the second with the fractures. This is the case with fractured reservoir rocks (Van Golf-Racht, 1982). Double porosity means that the permeabilities $\mathbf{K}_{p}$ and $\mathbf{K}_{f}$ of the pores and the fractures, respectively, are very different: $\mathbf{K}_{f} \gg \mathbf{K} p$.

This means in turn that there are two characteristic lengths $l$ and $l^{\prime}$ for the pores and the fractures, respectively, with $l / l^{\prime}=\varepsilon \ll 1$. 
At the same time the porosity of the fractures $n^{\prime}$ is smaller than that of pore $n$ : $n^{\prime}<n$.

We consider a reservoir of dimension $\mathrm{O}\left(l^{\prime \prime}\right), l^{\prime \prime} \gg l^{\prime}$ and we look for a macroscopic description of this reservoir, i.e., a description which is valid at the scale $l^{\prime \prime}$. In other words, we wish to obtain a macroscopic continuous equivalent medium to the actual finely heterogeneous one.

A large number of papers have been devoted to the seepage of fluids through rigid porous media with double or heterogeneous porosity. For recent works the reader will refer to Quintard and Whitaker (1987, 1988), Saez et al. (1989) and Levy (1990). But a few ones are devoted to such media with deformable matrix. Among them, let us recall the works by Barenblatt et al. (1960), Warren and Root (1963) and Wilson and Aifantis (1982). These approaches are conducted at the macroscopic scale, using phenomenological approach. A common characteristic is the assumption that the macroscopic description introduces two different pressure fields, i.e., the pressures in the pores and the fractures, respectively. It is also assumed in Barenblatt et al. (1960) and Warren and Root (1963) that the deformability of the solid only intervenes through the isotropic part of the solid stress, i.e., through the two pressure fields. A good review of the first two approaches and the corresponding solutions can be found in Van Golf-Racht (1982).

For the sake of simplicity we limit ourselves to elastic matrices with small deformations and the liquid filling the pores and the fractures will be considered as Newtonian and incompressible. In the same way the demonstrations of the existence and uniqueness of the solution of the boundary value problems to be solved at each step will be avoided. They are very often similar to those introduced for studying single porosity media and most of them can be found in Auriault (1991) or Levy and Sanchez-Palencia (1975). They all make use of variational formulations which are very useful for finding effective coefficient properties.

We use an homogenization technique to obtain the macroscopic description from the description at the pore and fracture scales. In Auriault (1991) it is shown both that the asymptotic developments method was the most powerful and that random and periodic media behave macroscopically in the same way, if we limit ourselves to determining macroscopic description structure. In the following pages, we therefore adopt the homogenization process for periodic structures where the results are the most rigourous and precise. The reader will refer to Auriault (1991) for the methodology.

It appears that the result, i.e. the macroscopic description, is quite sensitive to the ratio between the different scales $l / l^{\prime}$ and $l^{\prime} / l^{\prime \prime}$. In other words, there exist different macroscopic descriptions depending on the double-porosity medium under consideration. This is a new important point which was not mentioned before in the literature. We limit ourselves in this paper to the case $l^{\prime} / l^{\prime \prime}=\left(l / l^{\prime}\right)^{2}$, which exhibits a coupling between the flows through the pores and the fractures. Other cases will be examined in a subsequent Paper II. 
In a real reservoir a rather wide spectrum of sizes of pores and fractures can exist. It is in general not embarrassing since the different cases are separated by powers of $\varepsilon$. With $\varepsilon$ small, say $10^{-3}$, this gives for each case a wide range of possible values of $l$ and $l^{\prime} . l$ and $l^{\prime}$ do not need to be well defined. Let us nevertheless suppose very large spectra. The question is how to choose between all the possible modellings: all cases are in some sense present. It will be seen in Paper II that one of the modellings refered as Case 2 is the richest one. Since it includes all other modellings, Case 2 will appear as the good choice for such very large spectrum media, even if the separation of scales between the pores and the fractures is poor $\left(l / l^{\prime}\right.$ not small). But it must be noted that the Case 2 is the more intricated one!

We first present in Section 2 estimations concerning spacial and time scales and evaluate the dimensionless numbers entering the equations at the micro-level.

Then, in Section 3 the homogenization process is performed, which leads to the macroscopic description. The matrix material is assumed to be elastic and the fluid is viscous Newtonian. The main result is that there is only one macroscopic pressure field at the first order (and not two as in models available in literature) and that the macroscopic description has a similar structure to that of a classical single-porosity medium, but with different effective parameters and a symmetry broken.

Section 4 is aimed at investigating a particular case where the material constituting the solid part is assumed to be incompressible. This permits us to point out the difference with the classical, two-scale description (single porosity). For comparison, let us recall the single-porosity description as introduced by Biot (1941) from a phenomenological approach and in Auriault and Sanchez-Palencia (1977) by using the homogenization process for periodic structures. It can be written in the form:

$$
\begin{aligned}
& \nabla\left(\operatorname{ce}\left(\mathbf{u}_{s}\right)-\alpha p\right)=0, \\
& \nabla(\mathbf{K} \nabla p)=-\alpha \frac{\delta\left(\mathbf{e}\left(\mathbf{u}_{s}\right)\right)}{\delta t}-\beta \frac{\delta p}{\delta t} .
\end{aligned}
$$

This system relates the pore pressure $p$ and the displacement $\mathbf{u}_{s}$ of the matrix. $\mathbf{K}$ is the permeability and $\mathbf{e}$ the deformation. The system exhibits a symmetry: the same tensor $\alpha$ intervenes in the two equations.

\section{Estimations}

We consider a porous medium with double porosity, exhibiting a double spacial periodicity. At the pore scale, the period $\Omega$ has a characteristic length $l$, the solid occupies the domain $\Omega_{s}$ and the pores the domain $\Omega_{p}$, respectively. The boundary between the two is denoted $\Gamma$, see Figure 1. At the fracture scale, the period is $\Omega^{\prime}$ with a characteristic length $l^{\prime}$. The solid plus the pores occupies the domain $\Omega_{s p}^{\prime}$ and the fractures the domain $\Omega_{f}^{\prime}$. The boundary between the two is $\Gamma^{\prime}$, see Figure 2. It should be recalled that the fractured reservoir has a characteristic length $l^{\prime \prime}$. 
Let us consider a reservoir with $l^{\prime \prime}=10^{4} \mathrm{~m}$. Therefore, we have

$$
\frac{l^{\prime}}{l^{\prime \prime}}=2 \times 10^{-5}=o\left(\varepsilon^{2}\right)
$$

In the following, we investigate the case where $l^{\prime} / l^{\prime \prime}=\left(l / l^{\prime}\right)^{2}$. It should be noted that the ratio $l^{\prime} l l^{\prime \prime}$ is quite sensitive to the considered data. As an example, an other value of $l^{\prime \prime}$, say $l^{\prime \prime}=10^{2} \mathrm{~m}$, would lead to $l^{\prime} / l^{\prime \prime}=O\left(l / l^{\prime}\right)$. And, as it will be seen from the following analysis, the resulting macroscopic description would be different. We shall postpone such other similar cases to a later Paper II.

The three characteristic lengths $l, l^{\prime}$ and $l^{\prime \prime}$ introduce three dimensionless space variables and each unknown of a given boundary value problem a priori appears as a function of these three dimensionless space variables, among others, for example the time. We will rather use physical space variables, i.e., $\mathbf{x}, \mathbf{x}^{\prime}$ and $\mathbf{x}^{\prime \prime}$, with $\mathbf{x}=\varepsilon^{-3} \mathbf{x}^{\prime \prime}$, and $\mathbf{x}^{\prime}=\varepsilon^{-2} \mathbf{x}^{\prime \prime}$. Therefore, an unknown $\Phi$ appears as

$$
\Phi=\Phi\left(\mathbf{x}, \mathbf{x}^{\prime}, \mathbf{x}^{\prime \prime}, \ldots\right), \quad \mathbf{x}=\varepsilon^{-3} \mathbf{x}^{\prime \prime} \quad \text { and } \quad \mathbf{x}^{\prime}=\varepsilon^{-2} \mathbf{x}^{\prime \prime} .
$$

And the spacial periodicities of the porous medium induces the $\Omega$ and $\Omega^{\prime}$ periodicities of $\Phi$ with respect to $\mathbf{x}$ and $\mathbf{x}^{\prime}$, respectively. See Auriault (1991) for the equivalence of the existence of separated scales with (3), $\Phi \Omega$ and $\Omega^{\prime}$ periodic. The next step is to look for each unknown using a perturbation method, i.e. looking for $\Phi$ in the form of an asymptotic development with respect to the powers of $\varepsilon$ :

$$
\Phi=\Phi^{0}\left(\mathbf{x}, \mathbf{x}^{\prime}, \mathbf{x}^{\prime \prime}, \ldots\right)+\varepsilon \Phi^{1}\left(\mathbf{x}, \mathbf{x}^{\prime}, \mathbf{x}^{\prime \prime}, \ldots\right)+\cdots,
$$

$\Phi^{i} \Omega$ and $\Omega^{\prime}$ periodic.

\subsection{THE TWO TIME SCALES}

We focus on the descriptions at the micro-scales which show two characteristic times. It is given by the following set where the subscripts $s, p$ and $f$ stands for the solid, the fluid in the pores and the fluid in the fractures, respectively.

- In the solid matrix the equations are the quasi-static momentum balance

$$
\nabla \sigma_{s}=0,
$$

and the constitutive law (infinitesimal elasticity)

$$
\sigma_{s}=\mathbf{a e}\left(\mathbf{u}_{s}\right)
$$

where the stress $\sigma_{s}$ is related to the small deformation

$$
\mathbf{e}\left(\mathbf{u}_{s}\right)=1 / 2\left(\nabla+{ }^{t} \nabla\right) \mathbf{u}_{s}
$$

by the elastic tensor $\mathbf{a}$.

- In the fluid we have the quasi-static momentum balance

$$
\mu \Delta \mathbf{v}_{\alpha}-\nabla p_{\alpha}=0, \quad \sigma_{\alpha}=-p_{\alpha} I+2 \mu \mathbf{D},
$$


where $\mu$ is the viscosity, $\mathbf{v}$ the velocity, $p$ the pressure and $\mathbf{D}$ the rate of deformation: $\mathbf{D}=\mathbf{e}(\mathbf{v})$. The subscript $\alpha$ takes the values $p$ and $f$ in the pores and the fractures, respectively.

The incompressibility is written

$$
\nabla \mathbf{v}_{\alpha}=0, \quad \alpha=p, f .
$$

- The boundary conditions on $\Gamma$ are the continuity of the normal stresses and the velocities

$$
\sigma_{s} \mathbf{N}=\sigma_{p} \mathbf{N} \text { on } \Gamma \text {, }
$$

where $\mathbf{N}$ is the unit normal to $\Gamma$ and

$$
\mathbf{v}_{s}=\mathbf{v}_{p} \text { on } \Gamma \text {. }
$$

- The boundary conditions on $\Gamma^{\prime}$ will be evoked below.

Consider Equation (7), which introduces the dimensionless numbers

$$
Q_{\alpha}=\mu \frac{\Delta \mathbf{v}_{\alpha}}{\nabla p_{\alpha}}, \quad \alpha=p, f .
$$

The driving force in our problem is a macroscopic pressure gradient $\nabla p_{\alpha}=O\left(p / l^{\prime \prime}\right)$.

In the same time the velocity varies in the pores and the fractures, which gives for the viscous terms

$$
\mu \Delta \mathbf{v}_{p}=O\left(\mu \frac{\mathbf{v}_{p}}{l^{2}}\right), \quad \mu \Delta \mathbf{v}_{f}=O\left(\mu \frac{\mathbf{v}_{f}}{l^{\prime 2}}\right) .
$$

And from Equation (7) the viscous terms balance the pressure gradient

$$
\frac{p}{l^{\prime \prime}}=O\left(\mu \frac{\mathbf{v}_{f}}{l^{\prime 2}}\right)=O\left(\mu \frac{\mathbf{v}_{p}}{l^{2}}\right) .
$$

Now, due to the very different sizes, the flow through the pores and the fractures introduces two characteristic times $T_{p}$ and $T_{f}$, respectively. We define them by

$$
\mathbf{v}_{p}=\mathbf{u}_{p} / T_{p}, \quad \mathbf{v}_{f}=\mathbf{u}_{f} / T_{f},
$$

where $\mathbf{u}_{p}=\mathbf{u}_{f}$ is a reference displacement and where we assume $\mathbf{u}_{p}=\mathbf{u}_{f}=O\left(\mathbf{u}_{s}\right)$. Then from (11) we obtain

$$
\frac{T_{f}}{T_{p}}=O\left(\frac{l^{2}}{l^{\prime 2}}\right)=O\left(\varepsilon^{2}\right)
$$

In the same way as above for the different spacial scales, these two separated time scales introduce two dimensionless times and each unknown appears as a function of these two. We prefer to use physical time variables, i.e. $t$ and $T$ with $T=\varepsilon^{2} t$. $t$ and $T$ are well suited to describe the flows in the fractures and the pores, respectively. Finally, taking into account this new feature, an unknown $\Phi$ will be 
looked for in the form:

$$
\Phi=\Phi^{0}\left(\mathbf{x}, \mathbf{x}^{\prime}, \mathbf{x}^{\prime \prime}, t, T\right)+\varepsilon \Phi^{1}\left(\mathbf{x}, \mathbf{x}^{\prime}, \mathbf{x}^{\prime \prime}, t, T\right)+\cdots,
$$

$\mathbf{x}=\varepsilon^{-3} \mathbf{x}^{\prime \prime}, \quad \mathbf{x}^{\prime}=\varepsilon^{-2} \mathbf{x}^{\prime \prime}, \quad T=\varepsilon^{2} t, \Phi^{i}, \quad \Omega$ and $\Omega^{\prime}$ periodic. The writing (13) induces the choices of $\mathbf{x}^{\prime \prime}$ and $t$ as driving space and time variable, respectively. Therefore, equations are to be normalized by using $l^{\prime \prime}$ and $T_{f}$ as the characteristic length and time, respectively.

\subsection{NORMALIZATION}

The set introduced above for the micro-description exhibits dimensionless numbers and it is clear that these numbers must be estimated, using the powers of $\varepsilon$ as a measure, before introducing developments as (13) into the equations. The estimations are directly related to the problem under consideration. We now investigate such dimensionless numbers.

Firstly, we assume the macroscopic stresses in the solid part, the pores and the fractures to be of the same order of magnitude. Therefore, (9) is correctly normalized since the macroscopic point of view (13) has been adopted: $\mathbf{x}^{\prime \prime}$ is the driving space variable and $l^{\prime \prime}$ is then the right length to normalize.

Secondly, consider Equation (7) and the dimensionless number $Q_{\alpha}, \alpha=p, f$. By using $l^{\prime \prime}$ and $T_{f}$ to normalize as indicated by (13), we obtain

$$
Q_{p}=\frac{\mu \Delta \mathbf{v}_{p}}{\nabla p_{p}}=O\left(\frac{\mu \mathbf{u}_{p} l^{\prime \prime}}{l^{\prime 2} T_{f} p}\right)
$$

and with (11) and (12)

$$
Q_{p}=O\left(\frac{\mu \mathbf{u}_{p} l^{2} T_{p}}{l^{\prime \prime 2} T_{f} \mu \mathbf{u}_{p}}\right)=O\left(\frac{l^{2} T_{p}}{l^{\prime \prime 2} T_{f}}\right)=O\left(\varepsilon^{4}\right) .
$$

Using the same approach for the fractures leads to

$$
Q_{f}=\frac{\mu \Delta \mathbf{v}_{f}}{\nabla p_{f}}=O\left(\frac{\mu \mathbf{u}_{f} l^{\prime \prime}}{l^{\prime \prime 2} T_{f} p}\right)=O\left(\varepsilon^{4}\right) .
$$

Therefore, Equation (7) is formally normalized by

$$
\varepsilon^{4} \mu \Delta \mathbf{v}_{\alpha}-\nabla p_{\alpha}=0, \quad \alpha=p, f,
$$

and the corresponding constitutive laws are

$$
\sigma_{\alpha}=-p_{\alpha} \mathbf{I}+2 \varepsilon^{4} \mu \mathbf{D} .
$$

Thirdly, we investigate the boundary conditions on $\Gamma^{\prime}$, between the domains $\Omega_{s p}^{\prime}$ and $\Omega_{f}^{\prime}$. Due to the separation of scales, $l / l^{\prime}=\varepsilon \ll 1, \Omega_{s p}^{\prime}$ appears, at least at the first order as a porous medium immersed in a fluid $\Omega_{f}^{\prime}$. We can therefore use the results of Levy and Sanchez-Palencia (1975), which simply show the continuity of the 
pressure, the total normal stress and the relative fluxes to the solid matrix

$$
p_{p}=p_{f}, \quad\left(\left\langle\sigma_{s}\right\rangle_{\Omega}+\left\langle\sigma_{p}\right\rangle_{\Omega}\right) \mathbf{N}^{\prime}=\sigma_{f} \mathbf{N}^{\prime}, \quad \mathbf{v}_{f}-\left\langle\mathbf{v}_{s}\right\rangle_{\Omega}=\left\langle\mathbf{v}_{p}\right\rangle_{\Omega} \quad \text { on } \Gamma^{\prime},
$$

where $\mathbf{N}^{\prime}$ is a unit normal to $\Gamma^{\prime}$ and $\left\langle\sigma_{s}\right\rangle_{\Omega},\left\langle\mathbf{v}_{s}\right\rangle_{\Omega}$ and $\left\langle\mathbf{v}_{p}\right\rangle_{\Omega}$ are volume averages on $\Omega$, which are equal to surface averages and then are fluxes. $\langle\cdot\rangle=\int_{\Omega} \cdot \mathrm{d} \Omega$, where the quantity to be integrated is taken as zero-valued outside its domain of definition.

In conclusion the normalized set is written in the form

$$
\begin{aligned}
& \nabla \sigma_{s}=0 \\
& \sigma_{s}=\mathbf{a e}\left(\mathbf{u}_{s}\right) \quad \text { in } \Omega_{s}, \\
& \varepsilon^{4} \mu \Delta \mathbf{v}_{\alpha}-\nabla p_{\alpha}=0, \\
& \sigma_{\alpha}=-p_{\alpha} \mathbf{I}+2 \varepsilon^{4} \mu \mathbf{D}, \\
& \nabla \mathbf{v}_{\alpha}=0 \text { in } \Omega_{\alpha}, \\
& \sigma_{s} \mathbf{N}=\sigma_{p} \mathbf{N}, \\
& \mathbf{u}_{s}=\mathbf{u}_{p} \text { on } \Gamma, \\
& p_{p}=p_{f}, \\
& \left(\left\langle\sigma_{s}\right\rangle_{\Omega}+\left\langle\sigma_{p}\right\rangle_{\Omega}\right) \mathbf{N}^{\prime}=\sigma_{f} \mathbf{N}^{\prime}, \\
& \mathbf{v}_{f}-\left\langle\mathbf{v}_{s}\right\rangle_{\Omega}=\left\langle\mathbf{v}_{p}\right\rangle_{\Omega} \text { on } \Gamma^{\prime} .
\end{aligned}
$$

The unknowns are looked for in the form of asymptotic developments like (13), taking into account the fact that in $\Omega_{f}^{\prime}$ the solution is $\mathbf{x}$-independent, except in possible boundary layers along $\Gamma^{\prime}$, where velocities and pressures in the pores and in the fractures are matched

$$
\begin{aligned}
& \mathbf{u}_{s}=\mathbf{u}_{s}^{0}\left(\mathbf{x}, \mathbf{x}^{\prime}, \mathbf{x}^{\prime \prime}, t, T\right)+\varepsilon \mathbf{u}_{s}^{1}\left(\mathbf{x}, \mathbf{x}^{\prime}, \mathbf{x}^{\prime \prime}, t, T\right)+\ldots, \\
& \mathbf{u}_{p}=\mathbf{u}_{p}^{0}\left(\mathbf{x}, \mathbf{x}^{\prime}, \mathbf{x}^{\prime \prime}, t, T\right)+\varepsilon \mathbf{u}_{p}^{1}\left(\mathbf{x}, \mathbf{x}^{\prime}, \mathbf{x}^{\prime \prime}, t, T\right)+\ldots, \\
& p_{p}=p_{p}^{0}\left(\mathbf{x}, \mathbf{x}^{\prime}, \mathbf{x}^{\prime \prime}, t, T\right)+\varepsilon p_{p}^{1}\left(\mathbf{x}, \mathbf{x}^{\prime}, \mathbf{x}^{\prime \prime}, t, T\right)+\cdots, \\
& \mathbf{u}_{f}=\mathbf{u}_{f}^{0}\left(\mathbf{x}^{\prime}, \mathbf{x}^{\prime \prime}, t, T\right)+\varepsilon^{2} \mathbf{u}_{f}^{2}\left(\mathbf{x}^{\prime}, \mathbf{x}^{\prime \prime}, t, T\right)+\cdots, \\
& p_{f}=p_{f}^{0}\left(\mathbf{x}^{\prime}, \mathbf{x}^{\prime \prime}, t, T\right)+\varepsilon^{2} p_{f}^{2}\left(\mathbf{x}^{\prime}, \mathbf{x}^{\prime \prime}, t, T\right)+\cdots, \\
& \mathbf{x}=\varepsilon^{-3} \mathbf{x}^{\prime \prime}, \quad \mathbf{x}^{\prime}=\varepsilon^{-2} \mathbf{x}^{\prime \prime}, \quad T=\varepsilon^{2} t,
\end{aligned}
$$

$\mathbf{u}_{s}^{i}, \mathbf{u}_{p}^{i}, p_{p}^{1} \Omega$ and $\mathbf{\Omega}^{\prime}$ periodic and $\mathbf{u}_{f}^{i}, p_{f}^{i} \mathbf{\Omega}^{\prime}$ periodic. Due to the fact that $\mathbf{u}_{f}$ and $p_{f}$ are $\mathbf{x}$-independent their asymptotic developments are taken with respect to the powers of $\varepsilon^{2}$.

\section{Macroscopic Description}

Introducing the above developments in the set (14)-(23), we obtain successive boundary value problems to be solved on the periodic cell and concerning the 
different terms of the expansions. They are investigated in the Appendix and lead to the macroscopic equivalent description. This one is composed of a macroscopic bulk momentum balance and a macroscopic mass balance.

The macroscopic bulk momentum balance is (A.28)

$$
\nabla^{\prime \prime}\left(\mathbf{c}^{\prime \prime} \mathbf{e}^{\prime \prime}\left(\mathbf{u}_{s}^{0}\right)-\alpha^{\prime \prime} p^{0}\right)=0 .
$$

As expected, the space variable is $\mathbf{x}^{\prime \prime}$. It relates the two unknowns $\mathbf{u}_{s}^{0}\left(\mathbf{x}^{\prime \prime}, t, T\right)$ and $p^{0}\left(\mathbf{x}^{\prime \prime}, t, T\right)$, which represent the macroscopic displacement and the macroscopic fluid pressure. The structure of (24) is quite similar to the classical corresponding Biot Equation (1) for a single-pore system medium.

The macroscopic mass balance is (A.33)

$$
\nabla^{\prime \prime}\left(\left\langle\mathbf{v}_{f}^{0}\right\rangle_{\Omega^{\prime}}-n^{\prime} \mathbf{v}_{s}^{0}\right)=-\gamma^{\prime \prime} \frac{\delta \mathbf{e}^{\prime \prime}\left(\mathbf{u}_{s}^{0}\right)}{\delta t}-\beta^{\prime \prime} \frac{\delta p^{0}}{\delta t},
$$

and from (A.29)

$$
\left\langle\mathbf{v}_{f}^{0}\right\rangle_{\Omega^{\prime}}-n^{\prime} \mathbf{v}_{s}^{0}=-\mathbf{K}_{f} \nabla^{\prime \prime} p^{0},
$$

where $\left\langle\mathbf{v}_{f}^{0}\right\rangle_{\Omega^{\prime}}$ is the average of the fluid velocity in the fractures and $\mathbf{K}_{f}$ is the permeability tensor of the fractures.

Here again the structure of the result looks similar to that obtained by Biot for a single-pore system medium, see (2). The set (24)-(26) represents the macroscopic description of the investigated double-porosity medium. The variables are $\mathbf{u}_{s}^{0}, p_{s}^{0}$, and $\left\langle\mathbf{v}_{f}^{0}\right\rangle$. Despite the similarity with the classical consolidation for a single-pore system media, where $\alpha^{\prime \prime}=\gamma^{\prime \prime}$ (Auriault and Sanchez-Palencia, 1977), it can be expected that, due to the coupling between the pores and the fractures, the effective coefficients are dissimilar. In fact, a more fundamental fact occurs: the symmetry $\alpha^{\prime \prime}=\gamma^{\prime \prime}$ is broken and we have now in general $\gamma^{\prime \prime} \neq \alpha^{\prime \prime}$. This is shown in the next part for a particular case.

\section{Incompressible Elastic Material}

With a view to underlining the difference with the description of a single-porosity medium, let us consider a porous matrix made of incompressible elastic material. It is a classical assumption in soil mechanics. In this case the single-porosity medium gives $\alpha^{\prime \prime}=\gamma^{\prime \prime}$ (Auriault and Sanchez-Palencia, 1977).

Now, the behaviour law for the matric becomes

$$
\sigma_{s}=-p_{s} \mathbf{I}+\mathbf{a e}\left(\mathbf{u}_{s}\right),
$$

and we have to add the incompressibility condition $\nabla \mathbf{u}_{s}=0$.

Let us rapidly resume the approach adopted in Section 3, after having introduced the appropriate asymptotic development for the new unknown $p_{s}$. 
The reader will easily verify that the results of Section 3 and the Appendix are always valid until (A.24) included

$$
\begin{aligned}
& \mathbf{u}_{s}^{0}=\mathbf{u}_{s}^{0}\left(\mathbf{x}^{\prime \prime}, t, T\right), \quad \mathbf{u}_{s}^{1}=\mathbf{u}_{s}^{1}\left(\mathbf{x}^{\prime \prime}, t, T\right), \quad \mathbf{u}_{s}^{2}=\mathbf{u}_{s}^{2}\left(\mathbf{x}^{\prime}, \mathbf{x}^{\prime \prime}, t, T\right), \\
& p_{p}^{0}=p_{f}^{0}=p^{0}\left(\mathbf{x}^{\prime \prime}, t, T\right), \quad p_{p}^{1}=p_{p}^{1}\left(\mathbf{x}^{\prime}, \mathbf{x}^{\prime \prime}, t, T\right), \quad \mathbf{v}_{p}^{0}=\mathbf{v}_{s}^{0}=\frac{\delta \mathbf{u}_{s}^{0}}{\delta t}\left(\mathbf{x}^{\prime \prime}, t, T\right) .
\end{aligned}
$$

- The cell problem for $\mathbf{u}_{s}^{3}$ is changed and includes now the new unknown $p_{s}^{0}$ :

$$
\begin{aligned}
& \nabla \sigma_{s}^{0}=0, \quad \sigma_{s}^{0}=-p_{s}^{0} \mathbf{I}+\mathbf{a}\left(\mathbf{e}\left(\mathbf{u}_{s}^{3}\right)+\mathbf{e}^{\prime}\left(\mathbf{u}_{s}^{2}\right)+\mathbf{e}^{\prime \prime}\left(\mathbf{u}_{s}^{0}\right)\right), \\
& \nabla \mathbf{u}_{s}^{3}+\nabla^{\prime} \mathbf{u}_{s}^{0}+\nabla^{\prime \prime} \mathbf{u}_{s}^{0}=0, \quad \sigma_{s}^{0} \mathbf{N}=-p_{p}^{0} \mathbf{N} \quad \text { on } \Gamma .
\end{aligned}
$$

It is straightforward to obtain for $\mathbf{u}_{s}^{3}$, instead of (A.23),

$$
\mathbf{u}_{s}^{3}=\xi(\mathbf{x})\left(\mathbf{e}^{\prime}\left(\mathbf{u}_{s}^{2}\right)+\mathbf{e}^{\prime \prime}\left(\mathbf{u}_{s}^{0}\right)\right)+\hat{\mathbf{u}}_{s}^{3}\left(\mathbf{x}^{\prime}, \mathbf{x}^{\prime \prime}, t, T\right) .
$$

And we have

$$
p_{s}^{0}=p_{p}^{0}=p^{0}\left(\mathbf{x}^{\prime \prime}, t, T\right) .
$$

- The results of section A.4 are always valid:

$$
p_{p}^{1}=0 \quad \text { and } \quad \mathbf{v}_{p}^{1}=\mathbf{v}_{s}^{1}, \quad p_{p}^{2}=p_{p}^{2}\left(\mathbf{x}^{\prime}, \mathbf{x}^{\prime \prime}, t, T\right) .
$$

- The average over $\Omega$ of $\sigma_{s}^{0}$ (Section A.6) simplifies to

$$
\left\langle\sigma_{s}^{0}\right\rangle_{\Omega}=\mathbf{c}^{\prime}\left(\mathbf{e}^{\prime}\left(\mathbf{u}_{s}^{2}\right)+\mathbf{e}^{\prime \prime}\left(\mathbf{u}_{s}^{0}\right)\right)-(1-n) p^{0} \mathbf{I},
$$

i.e. with $\alpha^{\prime}=(1-n)$ I.

And due to the fact that $p^{0}$ is $\mathbf{x}$ - and $\mathbf{x}^{\prime}$-independent, the cell problem for $\mathbf{u}_{s}^{2}$ is now

$$
\begin{aligned}
& \nabla^{\prime}\left(\mathbf{c}^{\prime}\left(\mathbf{e}^{\prime}\left(\mathbf{u}_{s}^{2}\right)+\mathbf{e}^{\prime \prime}\left(\mathbf{u}_{s}^{0}\right)\right)\right)=0, \\
& \mathbf{c}^{\prime}\left(\mathbf{e}^{\prime}\left(\mathbf{u}_{s}^{2}\right)+\mathbf{e}^{\prime \prime}\left(\mathbf{u}_{s}^{0}\right)\right) \mathbf{N}^{\prime}=0 \text { on } \Gamma^{\prime},
\end{aligned}
$$

from which we deduce new expressions of (A.26) and (A.27) for $\mathbf{u}_{s}^{2}$ and $\mathbf{u}_{s}^{3}$, respectively:

$$
\begin{aligned}
& \mathbf{u}_{s}^{2}=\xi^{\prime}\left(\mathbf{x}^{\prime}\right) \mathbf{e}^{\prime \prime}\left(\mathbf{u}_{s}^{0}\right)+\hat{\mathbf{u}}_{s}^{2}\left(\mathbf{x}^{\prime \prime}, t, T\right), \\
& \mathbf{u}_{s}^{3}=\xi^{*}\left(\mathbf{x}, \mathbf{x}^{\prime}\right) \mathbf{e}^{\prime \prime}\left(\mathbf{u}_{s}^{0}\right)+\hat{\mathbf{u}}_{s}^{3}\left(\mathbf{x}^{\prime}, \mathbf{x}^{\prime \prime}, t, T\right) .
\end{aligned}
$$

Finally, the bulk momentum balance (A.28) is changed into:

$$
\nabla^{\prime \prime}\left(\mathbf{c}^{\prime \prime} \mathbf{e}^{\prime \prime}\left(\mathbf{u}_{s}^{0}\right)-p^{0} \mathbf{I}\right)=0,
$$

which is (24) with $\alpha^{\prime \prime}=\mathbf{I}$. We recover the structure of the classical bulk momentum balance for a single-porosity medium made of incompressible material.

- The macroscopic mass balance is also easy to obtain, following section A.7. We have always (A.30):

$$
n \nabla^{\prime \prime} \mathbf{v}_{p}^{0}+\nabla^{\prime}\left\langle\mathbf{v}_{p}^{2}\right\rangle_{\Omega}+\frac{1}{|\Omega|} \int_{\Gamma} \mathbf{v}_{s}^{3} \mathbf{N}_{p} \mathrm{~d} S=0
$$


But now a similar relation stands for the solid part:

$$
(1-n) \nabla^{\prime \prime} \mathbf{v}_{s}^{0}+(1-n) \nabla^{\prime} \mathbf{v}_{s}^{2}+\frac{1}{|\Omega|} \int_{\Gamma} \mathbf{v}_{s}^{3} \mathbf{N}_{s} \mathrm{~d} S=0 .
$$

Adding these two last relations member to member, together with the continuity of $\mathbf{v}^{3}$, on $\Gamma$, gives

$$
\nabla^{\prime}\left(\left\langle\mathbf{v}_{p}^{2}\right\rangle_{\Omega}+(1-n) \mathbf{v}_{s}^{2}\right)+\nabla^{\prime \prime} \mathbf{v}_{s}^{0}=0 .
$$

Relation (A.31) now becomes

$$
\nabla^{\prime}\left(\left\langle\mathbf{v}_{p}^{2}\right\rangle_{\Omega}-n \mathbf{v}_{s}^{2}\right)=-\nabla^{\prime \prime} \mathbf{v}_{s}^{0}-\nabla^{\prime} \mathbf{v}_{s}^{2} .
$$

Since we have again

$$
\int_{\Gamma^{\prime}} \mathbf{v}_{f}^{2} \mathbf{N}_{f}^{\prime} \mathrm{d} S=-\int_{\Omega^{\prime} s p}\left(\nabla^{\prime} \mathbf{v}_{s}^{2}+\nabla^{\prime}\left\langle\mathbf{v}_{p}^{2}\right\rangle\right) \mathrm{d} \Omega^{\prime},
$$

the new mass balance (25) is written in the form

$$
\nabla^{\prime \prime}\left(\left\langle\mathbf{v}_{f}^{0}\right\rangle_{\Omega^{\prime}}-n^{\prime} \mathbf{v}_{s}^{0}\right)=-\left(1-n\left(1-n^{\prime}\right)\right) \nabla^{\prime \prime} \mathbf{v}_{s}^{0},
$$

i.e. with $\gamma^{\prime \prime}=\left(1-n\left(1-n^{\prime}\right)\right) \mathbf{I}$ and $\beta^{\prime \prime}=0$.

This equation differs from the case of single porosity, where $\gamma^{\prime \prime}=\alpha^{\prime \prime}=1$ and $\beta^{\prime \prime}=0$, when the material constituting the porous matrix is incompressible. The symmetry is borken: $\gamma^{\prime \prime} \neq \alpha^{\prime \prime}$. This result emphasizes the difference between double and single porosity. Remark that when the pore porosity $n$ vanishes, i.e. when the medium becomes a single-porosity one, we recover the result $\gamma^{\prime \prime}=\alpha^{\prime \prime}=\mathbf{I}$. The case $n^{\prime}=0$ corresponds to a nonconnected fluid, where consolidation is absent and the theory developed has to be modified. The bulk medium behaves in this case as an incompressible elastic medium.

\section{Conclusion}

We have investigated the macroscopic description of a double porosity, deformable medium when the scale sizes are related by $l^{\prime} / l^{\prime \prime}=\left(l / l^{\prime}\right)^{2}$. The main results are:

- The macroscopic description exhibits only one pressure field.

- It looks like the single-porosity one with the pore plus matrix domain being macroscopically equivalent to an elastic monophasic medium. But effective coefficients are modified. In particular the $\gamma^{\prime \prime}=\alpha^{\prime \prime}$ symmetry is broken. And the elastic medium equivalent to the pore plus matrix domain does not behave like the empty matrix itself.

- The investigated case corrsponds to a full coupling of the flows through the pores and the fractures (see (A.32)).

- It is expected that other situations with different scale sizes would give different macroscopic description. This will be the subject of the Paper II. 
Appendix

Introducing the developments presented in Section 2 in the set (14)-(23), firstly we see that the corresponding developments for the stresses are written in the form

$$
\begin{aligned}
\sigma_{s} & =\varepsilon^{-3} \sigma_{s}^{-3}+\varepsilon^{-2} \sigma_{s}^{-2}+\varepsilon^{-1} \sigma_{s}^{-1}+\sigma_{s}^{0}+\ldots, \\
\sigma_{p} & =\sigma_{p}^{0}+\varepsilon \sigma_{p}^{1}+\varepsilon^{2} \sigma_{p}^{2}+\ldots \\
\sigma_{f} & =\sigma_{f}^{0}+\varepsilon^{2} \sigma_{f}^{2}+\ldots
\end{aligned}
$$

And noticing $\nabla, \nabla^{\prime}$ and $\nabla^{\prime \prime}$, the gradient operators with respect to $\mathbf{x}, \mathbf{x}^{\prime}$ and $\mathbf{x}^{\prime \prime}$, respectively, and adopting similar notations for other operators, we obtain successively by equating like powers of $\varepsilon$ :

In the solid part

$$
\begin{aligned}
& \sigma_{s}^{-3}=\mathbf{a e}\left(\mathbf{u}_{s}^{0}\right), \\
& \sigma_{s}^{-2}=\mathbf{a}\left(\mathbf{e}\left(\mathbf{u}_{s}^{1}\right)+\mathbf{e}^{\prime}\left(\mathbf{u}_{s}^{0}\right)\right), \\
& \sigma_{s}^{-1}=\mathbf{a}\left(\mathbf{e}\left(\mathbf{u}_{s}^{2}\right)+\mathbf{e}^{\prime}\left(\mathbf{u}_{s}^{1}\right)\right), \\
& \sigma_{s}^{0}=\mathbf{a}\left(\mathbf{e}\left(\mathbf{u}_{s}^{3}\right)+\mathbf{e}^{\prime}\left(\mathbf{u}_{s}^{2}\right)+\mathbf{e}^{\prime \prime}\left(\mathbf{u}_{s}^{0}\right)\right), \\
& \sigma_{s}^{1}=\mathbf{a}\left(\mathbf{e}\left(\mathbf{u}_{s}^{4}\right)+\mathbf{e}^{\prime}\left(\mathbf{u}_{s}^{3}\right)+\mathbf{e}^{\prime \prime}\left(\mathbf{u}_{s}^{1}\right)\right) \ldots \\
& \nabla \sigma_{s}^{-3}=0, \quad \nabla \sigma_{s}^{-2}+\nabla^{\prime} \sigma_{s}^{-3}=0, \quad \nabla \sigma_{s}^{-1}+\nabla^{\prime} \sigma_{s}^{-2}=0, \\
& \nabla \sigma_{s}^{0}+\nabla^{\prime} \sigma_{s}^{-1}+\nabla^{\prime \prime} \sigma_{s}^{-3}=0, \quad \nabla \sigma_{s}^{1}+\nabla^{\prime} \sigma_{s}^{0}+\nabla^{\prime \prime} \sigma_{s}^{-2}=0, \\
& \nabla \sigma_{s}^{2}+\nabla^{\prime} \sigma_{s}^{1}+\nabla^{\prime \prime} \sigma_{s}^{-1}=0, \quad \nabla \sigma_{s}^{3}+\nabla^{\prime} \sigma_{s}^{2}+\nabla^{\prime \prime} \sigma_{s}^{0}=0 \ldots
\end{aligned}
$$

In the pores

$$
\begin{aligned}
& \mathbf{v}_{p}^{0}=\frac{\partial \mathbf{u}_{p}^{0}}{\partial t}, \quad \mathbf{v}_{p}^{1}=\frac{\partial \mathbf{u}_{p}^{1}}{\partial t}, \quad \mathbf{v}_{p}^{2}=\frac{\partial \mathbf{u}_{p}^{2}}{\partial t}+\frac{\partial \mathbf{u}_{p}^{0}}{\partial T} \ldots \\
& \sigma_{p}^{0}=-p_{p}^{0} \mathbf{I}, \\
& \sigma_{p}^{1}=-p_{p}^{1} \mathbf{I}+2 \mu \mathbf{e}\left(\mathbf{v}_{p}^{0}\right), \\
& \sigma_{p}^{2}=-p_{p}^{2} \mathbf{I}+2 \mu\left(\mathbf{e}\left(\mathbf{v}_{p}^{1}\right)+\mathbf{e}^{\prime}\left(\mathbf{v}_{p}^{0}\right)\right), \\
& \sigma_{p}^{3}=-p_{p}^{3} \mathbf{I}+2 \mu\left(\mathbf{e}\left(\mathbf{v}_{p}^{2}\right)+\mathbf{e}^{\prime}\left(\mathbf{v}_{p}^{1}\right)\right) \ldots \\
& \nabla \sigma_{p}^{0}=0, \quad \nabla \sigma_{p}^{1}+\nabla^{\prime} \sigma_{p}^{0}=0, \\
& \nabla \sigma_{p}^{2}+\nabla^{\prime} \sigma_{p}^{1}=0, \quad \nabla \sigma_{p}^{3}+\nabla^{\prime} \sigma_{p}^{2}+\nabla^{\prime \prime} \sigma_{p}^{0}=0 \ldots \\
& \nabla \mathbf{v}_{p}^{0}=0, \quad \nabla \mathbf{v}_{p}^{1}+\nabla^{\prime} \mathbf{v}_{p}^{0}=0, \\
& \nabla \mathbf{v}_{p}^{2}+\nabla^{\prime} \mathbf{v}_{p}^{1}=0, \quad \nabla \mathbf{v}_{p}^{3}+\nabla^{\prime} \mathbf{v}_{p}^{2}+\nabla^{\prime \prime} \mathbf{v}_{p}^{0}=0 \ldots
\end{aligned}
$$


In the fractures

$$
\begin{array}{ll}
\mathbf{v}_{f}^{0}=\frac{\delta \mathbf{u}_{f}^{0}}{\delta t}, & \mathbf{v}_{f}^{2}=\frac{\delta \mathbf{u}_{f}^{2}}{\delta t}+\frac{\delta \mathbf{u}_{f}^{0}}{\delta T} \ldots \\
\sigma_{f}^{0}=-p_{f}^{0} \mathbf{l}, & \sigma_{f}^{2}=-p_{f}^{2} \mathbf{I}+2 \mu \mathbf{e}^{\prime}\left(\mathbf{v}_{f}^{0}\right) \ldots \\
\nabla^{\prime} \sigma_{f}^{0}=0, & \nabla^{\prime} \sigma_{f}^{2}+\nabla^{\prime \prime} \sigma_{f}^{0}=0, \quad \nabla^{\prime} \sigma_{f}^{4}+\nabla^{\prime \prime} \sigma_{f}^{2}=0 \ldots \\
\nabla^{\prime} \mathbf{v}_{f}^{0}=0, & \nabla^{\prime} \mathbf{v}_{f}^{2}+\nabla^{\prime \prime} \mathbf{v}_{f}^{0}=0 \ldots
\end{array}
$$

On $\Gamma$

$$
\begin{aligned}
& \sigma_{s}^{-3} \mathbf{N}=0, \quad \sigma_{s}^{-2} \mathbf{N}=0, \quad \sigma_{s}^{-1} \mathbf{N}=0, \\
& \sigma_{s}^{0} \mathbf{N}=\sigma_{p}^{0} \mathbf{N}, \quad \sigma_{s}^{1} \mathbf{N}=\sigma_{p}^{1} \mathbf{N}, \\
& \sigma_{s}^{2} \mathbf{N}=\sigma_{p}^{2} \mathbf{N}, \quad \sigma_{s}^{3} \mathbf{N}=\sigma_{p}^{3} \mathbf{N} \ldots \\
& \mathbf{v}_{s}^{0}=\mathbf{v}_{p}^{0}, \quad \mathbf{v}_{s}^{1}=\mathbf{v}_{p}^{1}, \quad \mathbf{v}_{s}^{2}=\mathbf{v}_{p}^{2}, \quad \mathbf{v}_{s}^{3}=\mathbf{v}_{p}^{3} \ldots \\
&
\end{aligned}
$$

On $\Gamma^{\prime}$

$$
\begin{aligned}
& p_{p}^{0}=p_{f}^{0}, \quad p_{p}^{1}=0, \quad p_{p}^{2}=p_{f}^{2} \ldots \\
& \left\langle\sigma_{s}^{-3}\right\rangle_{\Omega} \mathbf{N}^{\prime}=0, \quad\left\langle\sigma_{s}^{-2}\right\rangle_{\Omega} \mathbf{N}^{\prime}=0, \quad\left\langle\sigma_{s}^{-1}\right\rangle_{\Omega} \mathbf{N}^{\prime}=0, \\
& \left(\left\langle\sigma_{s}^{0}\right\rangle_{\Omega}+\left\langle\sigma_{p}^{0}\right\rangle_{\Omega}\right) \mathbf{N}^{\prime}=\sigma_{f}^{0} \mathbf{N}^{\prime}, \\
& \left(\left\langle\sigma_{s}^{1}\right\rangle_{\Omega}+\left\langle\sigma_{p}^{1}\right\rangle_{\Omega}\right) \mathbf{N}^{\prime}=0 \\
& \left(\left\langle\sigma_{s}^{2}\right\rangle_{\Omega}+\left\langle\sigma_{p}^{2}\right\rangle_{\Omega}\right) \mathbf{N}^{\prime}=\sigma_{f}^{2} \mathbf{N}^{\prime} \ldots \\
& \mathbf{v}_{f}^{0}-\left\langle\mathbf{v}_{s}^{0}\right\rangle_{\Omega}=\left\langle\mathbf{v}_{p}^{0}\right\rangle_{\Omega}, \\
& -\left\langle\mathbf{v}_{s}^{1}\right\rangle_{\Omega}=\left\langle\mathbf{v}_{p}^{1}\right\rangle_{\Omega}, \\
& \left.\mathbf{v}_{f}^{2}-\left\langle\mathbf{v}_{s}^{2}\right\rangle_{\Omega}\right)=\left\langle\mathbf{v}_{p}^{2}\right\rangle_{\Omega} \ldots
\end{aligned}
$$

Beginning by the lower orders, we now extract from (A.1)-(A.16) boundary value problems defined in the solid domain, the pore domain, the fracture domain, the solid plus pore domain or the bulk domain.

\section{A.1. WE FIRST CONSIDER THE SOLID DOMAIN}

- Equation (A.3a) with (A.2a) and (A.12a) is an homogeneous problem for $\mathbf{u}_{s}^{0}$ $\Omega$ periodic, with respect to the variable $\mathbf{x}$. It gives $\mathbf{u}_{s}^{0}$ as $\mathbf{x}$-independent:

$$
\mathbf{u}_{s}^{0}=\mathbf{u}_{s}^{0}\left(\mathbf{x}^{\prime}, \mathbf{x}^{\prime \prime}, t, T\right) \text { and } \sigma_{s}^{-3}=0 .
$$

- Equation (A.3b) therefore simplifies in $\nabla \sigma_{s}^{-2}=0$.

With (A.2b) and (A.12b) we obtain a linear problem for $\mathbf{u}_{s}^{1} \Omega$ periodic, as a function of $\mathbf{e}^{\prime}\left(\mathbf{u}_{s}^{0}\right)$ :

$$
\mathbf{u}_{s}^{1}=\xi(\mathbf{x}) \mathbf{e}^{\prime}\left(\mathbf{u}_{s}^{0}\right)+\hat{\mathbf{u}}_{s}^{1}\left(\mathbf{x}^{\prime}, \mathbf{x}^{\prime \prime}, t, T\right),
$$


where $\xi$ is a third-order tensor well defined by the boundary value problem and $\mathbf{u}_{s}^{1}$ an arbitrary vector $\mathbf{x}$-independent.

- Consider Equation (A.3c). Taking the volume average over $\Omega$, using the periodicity of $\sigma_{s}^{-1}$ together with (A.12c) leads to:

$$
\nabla^{\prime}\left\langle\sigma_{s}^{-2}\right\rangle_{\Omega}=0,
$$

where the averaged stress is given by

$$
\left\langle\sigma_{s}^{-2}\right\rangle_{\Omega}=\mathbf{c}^{\prime} \mathbf{e}^{\prime}\left(\mathbf{u}_{s}^{0}\right), \quad \mathbf{c}^{\prime}=\langle\mathbf{a}(\mathbf{I}+\mathbf{e}(\xi))\rangle_{\Omega} .
$$

We know, see Auriault and Sanchez-Palencia (1977), that $\mathbf{c}^{\prime}$ is an elastic tensor. Equation (A.18) and (A.15b) is therefore a well posed problem for $\mathbf{u}_{s}^{0} \Omega^{\prime}$ periodic. Since it is homogeneous, it is straightforward to obtain $\mathbf{u}_{s}^{0}=\mathbf{u}_{s}^{0}\left(\mathbf{x}^{\prime \prime}, t, T\right)$.

Therefore, $\mathbf{u}_{s}^{1}$ appears as $\mathbf{x}$-independent:

$$
\mathbf{u}_{s}^{1}=\mathbf{u}_{s}^{1}\left(\mathbf{x}^{\prime}, \mathbf{x}^{\prime \prime}, t, T\right) \quad \text { and } \sigma_{s}^{-2}=0 .
$$

And (A.3c) reduces to $\nabla \sigma_{s}^{-1}=0$.

Using the boundary condition (A.12c) on $\Gamma, \mathbf{u}_{s}^{2}$ appears as a linear vectorial function of $\mathbf{e}^{\prime}\left(\mathbf{u}_{s}^{1}\right)$, similar to (A.17):

$$
\mathbf{u}_{s}^{2}=\xi(\mathbf{x}) \mathbf{e}^{\prime}\left(\mathbf{u}_{s}^{1}\right)+\hat{\mathbf{u}}_{s}^{2}\left(\mathbf{x}^{\prime}, \mathbf{x}^{\prime \prime}, t, T\right) .
$$

\section{A.2. WE NOW TURN OUR ATTENTION TO THE PORE AND FRACTURE DOMAINS}

- Equation (A.6a) with (A.5a) gives $p_{p}^{0} \mathbf{x}$-independent:

$$
p_{p}^{0}=p_{p}^{0}\left(\mathbf{x}^{\prime}, \mathbf{x}^{\prime \prime}, t, T\right) \text {. }
$$

- The following order gives $\mathbf{v}_{p}^{0}$ and $p_{p}^{1}, \Omega$ periodic, as linear functions of $\nabla^{\prime} p_{p}^{0}$ through the classical boundary value problem (A.6b), (A.7a) with the adherence condition (A.13a) on $\Gamma \cdot \mathbf{v}_{s}^{0}$ being $\mathbf{x}$-independent, we obtain (for a more detailed explanation see Auriault (1991))

$$
\mathbf{v}_{p}^{0}-\mathbf{v}_{s}^{0}=-\mathbf{k}_{p}(\mathbf{x}) \nabla^{\prime} p_{p}^{0}, \quad p_{p}^{1}=\tau \nabla^{\prime} p_{p}^{0}+\hat{p}_{p}^{1}\left(\mathbf{x}^{\prime}, \mathbf{x}^{\prime \prime}, t, T\right) .
$$

where the tensor $\mathbf{k}_{p}$ and the vector $\tau$ are well defined by the boundary value problem, and $\hat{p}_{p}^{1}$ is an added $\mathbf{x}$-independent arbitrary function.

- Considering (A.13b), where $\mathbf{v}_{s}^{1}$ is $\mathbf{x}$-independent and integrating (A.7b) on $\Omega$ leads to $\nabla^{\prime}\left\langle\mathbf{v}_{p}^{0}\right\rangle_{\Omega}=0$.

Using now (41.a), where $\mathbf{v}_{s}^{0}$ is $\mathbf{x}^{\prime}$-independent gives in turn

$$
\nabla^{\prime}\left(\mathbf{K}_{p} \nabla^{\prime} p_{p}^{0}\right)=0, \quad \mathbf{K}_{p}=\left\langle\mathbf{K}_{p}\right\rangle_{\Omega},
$$

where $\mathbf{K}_{p}$ is a classical permeability tensor, symmetrical and positive.

Equation (A.20a) with (A.9a) restricts the expression of the fracture pressure to $p_{f}^{0}=p_{f}^{0}\left(\mathbf{x}^{\prime \prime}, t, T\right)$. 
Therefore Equation (A.21), defined in $\Omega_{s p}^{\prime}$ with the boundary condition (A.14a), gives the following result, $p_{f}^{0}$ being $\mathbf{x}^{\prime}$-independent: $p_{p}^{0}=p_{f}^{0}=p^{0}\left(\mathbf{x}^{\prime \prime}, t, T\right)$.

And (A.20a) reduces to

$$
\mathbf{v}_{p}^{0}=\mathbf{v}_{s}^{0}=\frac{\partial \mathbf{u}_{s}^{0}}{\partial t}\left(\mathbf{x}^{\prime \prime}, t, T\right)
$$

which means that, at the time scale $T_{f}$, there is no relative movement between the solid part and the fluid in the pore. We deduce also from (A.20b) that $p_{p}^{1}=p_{p}^{1}\left(\mathbf{x}^{\prime}, \mathbf{x}^{\prime \prime}, t, T\right)$.

\section{A.3. WE RETURN TO THE POROUS MATRIX}

- Taking into account the results above, Equation (A.3d) reduces to $\nabla \sigma_{s}^{0}+\nabla^{\prime} \sigma_{s}^{-1}=0$.

Using the boundary condition (A.12d) where $p_{p}^{0}$ is $\mathbf{x}$-independent and taking the average over $\Omega$ gives

$$
\nabla^{\prime}\left\langle\sigma_{s}^{-1}\right\rangle_{\Omega}=0
$$

where $\left\langle\sigma_{s}^{-1}\right\rangle$, with (A.2c) and the expression (A.19) of $\mathbf{u}_{s}^{2}$ is written

$$
\left\langle\sigma_{s}^{-1}\right\rangle_{\Omega}=\mathbf{c}^{\prime} \mathbf{e}^{\prime}\left(\mathbf{u}_{s}^{1}\right) \text {. }
$$

$\mathbf{c}^{\prime}$ is the elastic tensor previously introduced.

With the boundary condition (A.15c) on $\Gamma^{\prime},($ A.22) and (A.23) form an homogeneous set for the unknown $\mathbf{u}_{s}^{1}$. Therefore we obtain $\mathbf{u}_{s}^{1}=\mathbf{u}_{s}^{1}\left(\mathbf{x}^{\prime \prime}, t, T\right)$, from which (A.19) reduces to $\mathbf{u}_{s}^{2}=\mathbf{u}_{s}^{2}\left(\mathbf{x}^{\prime}, \mathbf{x}^{\prime \prime}, t, T\right)$, and as a consequence we have $\sigma_{s}^{-1}=0$.

- We now see that (A.3d) is restricted to $\nabla \sigma_{s}^{0}=0$, with $\sigma_{s}^{0}$ given by (A.2d) and the boundary condition (A.12d) on $\Gamma: \sigma_{s}^{0} \mathbf{N}=-p_{p}^{0} \mathbf{N}$.

As a consequence, $\mathbf{u}_{s}^{3}$ appears as a linear vectorial function of both $\mathbf{e}^{\prime}\left(\mathbf{u}_{s}^{2}\right)+\mathbf{e}^{\prime \prime}\left(\mathbf{u}_{s}^{0}\right)$ and $p_{p}^{0}$ :

$$
\mathbf{u}_{s}^{3}=\xi(\mathbf{x})\left(\mathbf{e}^{\prime}\left(\mathbf{u}_{s}^{2}\right)+\mathbf{e}^{\prime \prime}\left(\mathbf{u}_{s}^{0}\right)\right)-\eta(\mathbf{x}) p_{p}^{0}+\hat{\mathbf{u}}_{s}^{3}\left(\mathbf{x}^{\prime}, \mathbf{x}^{\prime \prime}, t, T\right),
$$

where $\xi$ was defined above and $\eta$ is a new, $\mathbf{x}$-dependent vector. They both can be obtained by solving the boundary value problem.

\section{A.4. WE COME BACK AGAIN TO THE PORES}

$-\mathbf{v}_{p}^{1}$ is given by (A.6c), (A.5c) where $\mathbf{e}^{\prime}\left(\mathbf{v}_{p}^{0}\right)$ cancels out and the condition (A.13b) on $\Gamma$. The problem is similar to that for $v_{p}^{0}$ and the solution is written:

$$
\mathbf{v}_{p}^{1}-\mathbf{v}_{s}^{1}=-\mathbf{k}_{p}(\mathbf{x}) \nabla^{\prime} p_{p}^{1}, \quad p_{p}^{2}=\tau \nabla^{\prime} p_{p}^{1}+p_{p}^{2}\left(\mathbf{x}^{\prime}, \mathbf{x}^{\prime \prime}, t, T\right) .
$$


- The mass balance $(\mathrm{A} .7 \mathrm{c})$, integrated on $\Omega$, with the help of the condition $(\mathrm{A} .13 \mathrm{c})$ on $\Gamma$ :

$$
\mathbf{v}_{p}^{2}=\mathbf{v}_{s}^{2}=\frac{\partial \mathbf{u}_{p}^{2}}{\partial t}+\frac{\partial \mathbf{u}_{p}^{0}}{\partial T},
$$

where the right-hand term is $\mathbf{x}$-independent, gives $\nabla^{\prime}\left\langle\mathbf{v}_{p}^{1}\right\rangle_{\Omega}=0$. Using (A.25a) and the fact that $\mathbf{v}_{s}^{1}$ is $\mathbf{x}^{\prime}$-independent, we obtain $\nabla^{\prime}\left(\mathbf{K}_{p} \nabla^{\prime} p_{p}^{1}\right)=0$. This equation together with (A.14b) on $\Gamma$ is an homogeneous problem for $p_{p}^{1}$ and $p_{p}^{1}=0$, and therefore

$$
\mathbf{v}_{p}^{1}=\mathbf{v}_{s}^{1}, \quad p_{p}^{2}=p_{p}^{2}\left(\mathbf{x}^{\prime}, \mathbf{x}^{\prime \prime}, t, T\right) .
$$

\section{A.5. UP TO NOW THE RESULTS ARE}

$$
\begin{aligned}
& \mathbf{u}_{s}^{0}=\mathbf{u}_{s}^{0}\left(\mathbf{x}^{\prime \prime}, t, T\right), \quad \mathbf{u}_{s}^{1}=\mathbf{u}_{s}^{1}\left(\mathbf{x}^{\prime \prime}, t, T\right), \quad \mathbf{u}_{s}^{2}=\mathbf{u}_{s}^{2}\left(\mathbf{x}^{\prime}, \mathbf{x}^{\prime \prime}, t, T\right), \\
& \mathbf{u}_{s}^{3}=\xi(\mathbf{x})\left(\mathbf{e}^{\prime}\left(\mathbf{u}_{s}^{2}\right)+\mathbf{e}^{\prime \prime}\left(\mathbf{u}_{s}^{0}\right)\right)-\eta(\mathbf{x}) p_{p}^{0}+\hat{\mathbf{u}}_{s}^{3}\left(\mathbf{x}^{\prime}, \mathbf{x}^{\prime \prime}, t, T\right), \\
& p_{p}^{0}=p_{f}^{0}=p^{0}\left(\mathbf{x}^{\prime \prime}, t, T\right), \quad p_{p}^{1}=0, \quad p_{p}^{2}=p_{p}^{2}\left(\mathbf{x}^{\prime}, \mathbf{x}^{\prime \prime}, t, T\right), \\
& \mathbf{v}_{p}^{0}=\mathbf{v}_{s}^{0}=\frac{\partial \mathbf{u}_{s}^{0}}{\partial t}\left(\mathbf{x}^{\prime \prime}, t, T\right), \quad \mathbf{v}_{p}^{1}=\mathbf{v}_{s}^{1}=\frac{\partial \mathbf{u}_{s}^{1}}{\partial t}\left(\mathbf{x}^{\prime \prime}, t, T\right) .
\end{aligned}
$$

\section{A.6. WE ARE NOW IN A POSITION TO OBTAIN A BULK MOMENTUM BALANCE}

- Equation (A.3e) simplifies to $\nabla \sigma_{s}^{1}+\nabla^{\prime} \sigma_{s}^{0}=0$.

Averaging over $\Omega$, with (A.12e) on $\Gamma: \sigma_{s}^{1} \mathbf{N}=\sigma_{p}^{1} \mathbf{N}=0$, gives $\nabla^{\prime}\left\langle\sigma_{s}^{0}\right\rangle_{\Omega}=0$.

This last equation can be rewritten by using the fact that $p^{0}$ is $\mathbf{x}^{\prime}$-independent

$$
\nabla^{\prime}\left(\left\langle\sigma_{s}^{0}\right\rangle_{\Omega}-n p^{0} \mathbf{I}\right)=0,
$$

where $\left\langle\sigma_{s}^{0}\right\rangle_{\Omega}$ is obtained from (A.2d) and the above results (A.24) concerning $\mathbf{u}_{s}^{3}$

$$
\left\langle\sigma_{s}^{0}\right\rangle_{\Omega}=\mathbf{c}^{\prime}\left(\mathbf{e}^{\prime}\left(\mathbf{u}_{s}^{2}\right)+\mathbf{e}^{\prime \prime}\left(\mathbf{u}_{s}^{0}\right)\right)-\alpha^{\prime} p^{0} .
$$

With the condition (A.15d) on $\Gamma^{\prime}$

$$
\left(\left\langle\sigma_{s}^{0}\right\rangle_{\Omega}-n p^{0} \mathbf{I}\right) \mathbf{N}^{\prime}=-p^{0} \mathbf{N}^{\prime},
$$

we obtain $\mathbf{u}_{s}^{2}$ in the form of a linear vectorial function of $\mathbf{e}^{\prime \prime}\left(\mathbf{u}_{s}^{0}\right)$ and $p^{0}$ :

$$
\mathbf{u}_{s}^{2}=\xi^{\prime}\left(\mathbf{x}^{\prime}\right) \mathbf{e}^{\prime \prime}\left(\mathbf{u}_{s}^{0}\right)-\eta^{\prime}\left(\mathbf{x}^{\prime}\right) p_{p}^{0}+\hat{\mathbf{u}}_{s}^{2}\left(\mathbf{x}^{\prime \prime}, t, T\right),
$$

where $\hat{\mathbf{u}}_{s}^{2}$ is an arbitrary added vector. And the expression (A.24) for $\mathbf{u}_{s}^{3}$ becomes

$$
\mathbf{u}_{s}^{3}=\xi^{*}\left(\mathbf{x}, \mathbf{x}^{\prime}\right) \mathbf{e}^{\prime \prime}\left(\mathbf{u}_{s}^{0}\right)-v^{\prime}\left(\mathbf{x}, \mathbf{x}^{\prime}\right) p_{p}^{0}+\hat{\mathbf{u}}_{s}^{3}\left(\mathbf{x}^{\prime}, \mathbf{x}^{\prime \prime}, t, T\right) .
$$

- Now consider (A.3g) and (A.6d)

$$
\nabla \sigma_{s}^{3}+\nabla^{\prime} \sigma_{s}^{2}+\nabla^{\prime \prime} \sigma_{s}^{0}=0, \quad \nabla \sigma_{p}^{3}+\nabla^{\prime} \sigma_{p}^{2}+\nabla^{\prime \prime} \sigma_{p}^{0}=0 .
$$


Averaging on $\Omega_{s}$ and $\Omega_{p}$, respectively, and using the condition (A.12g) on $\Gamma$ gives

$$
\nabla^{\prime}\left\langle\sigma_{s}^{2}+\sigma_{p}^{2}\right\rangle_{\Omega}+\nabla^{\prime \prime}\left\langle\sigma_{s}^{0}+\sigma_{p}^{0}\right\rangle_{\Omega}=0 .
$$

We join this equation with (A.10b): $\nabla^{\prime} \sigma_{f}^{2}+\nabla^{\prime \prime} \sigma_{f}^{0}=0$.

Averaging these last two on $\Omega_{s p}^{\prime}$ and $\Omega_{f}^{\prime}$, respectively, and using the condition (A.15f) on $\Gamma^{\prime}$ now gives

$$
\nabla^{\prime \prime}\left\langle\sigma_{f}^{0}\right\rangle_{\Omega^{\prime}}+\nabla^{\prime \prime}\left\langle\left\langle\sigma_{s}^{0}+\sigma_{p}^{0}\right\rangle_{\Omega \Omega^{\prime}}=0,\right.
$$

where

$$
\begin{aligned}
\left.\| \sigma_{s}^{0}\right\rangle_{\Omega \Omega^{\prime}} & =\left\langle\mathbf{c}^{\prime}\left(\mathbf{e}^{\prime}\left(\mathbf{u}_{s}^{2}\right)+\mathbf{e}^{\prime \prime}\left(\mathbf{u}_{s}^{0}\right)\right)-\alpha^{\prime} p^{0}>_{\boldsymbol{\Omega}^{\prime}}\right. \\
& =\mathbf{c}^{\prime \prime} \mathbf{e}^{\prime \prime}\left(\mathbf{u}_{s}^{0}\right)-\alpha^{*} p^{0} .
\end{aligned}
$$

Finally

$$
\nabla^{\prime \prime}\left(\mathbf{c}^{\prime \prime} \mathbf{e}^{\prime \prime}\left(\mathbf{u}_{s}^{0}\right)-\alpha^{\prime \prime} p^{0}=0\right.
$$

which represents a macroscopic momentum balance for the bulk medium.

\section{A.7. WE LOOK FOR A MACROSCOPIC MASS BALANCE}

- The velocity $\mathbf{v}_{f}^{0}$ in the fractures is given by (A.10b) with (A.9b) and (A.11a):

$$
\mu \Delta^{\prime} \mathbf{v}_{f}^{0}-\nabla^{\prime} p_{f}^{2}-\nabla^{\prime \prime} p^{0}=0, \quad \nabla^{\prime} \mathbf{v}_{f}^{0}=0,
$$

together with the boundary condition (A.16a) on $\Gamma^{\prime}$, which reduces to $\mathbf{v}_{f}^{0}=\mathbf{v}_{s}^{0}$. It is again a classical problem for the flow through a porous medium

$$
\mathbf{v}_{f}^{0}-\mathbf{v}_{s}^{0}=-\mathbf{k}_{f}\left(\mathbf{x}^{\prime}\right) \nabla^{\prime \prime} p^{0}
$$

where $\mathbf{k}_{f}$ is a second-order tensor well defined by the boundary value problem.

- Taking into account all the above results, (A.6d) and (A.7c) becomes respectively

$$
\mu \Delta \mathbf{v}_{p}^{2}-\nabla p_{p}^{3}-\nabla^{\prime} p_{p}^{2}-\nabla^{\prime \prime} p^{0}=0, \quad \nabla \mathbf{v}_{p}^{2}=0 .
$$

Since we have (A.13c), i.e. $\mathbf{v}_{p}^{2}=\mathbf{v}_{s}^{2}$ on $\Gamma$, where $\mathbf{v}_{s}^{2}$ is $\mathbf{x}$-independent as well as $\nabla^{\prime} p_{p}^{2}$ and $\nabla^{\prime \prime} p^{0}$, the solution for $\mathbf{v}_{p}^{2}$ is written in the form

$$
\mathbf{v}_{p}^{2}-\mathbf{v}_{s}^{2}=-\mathbf{k}_{p}(\mathbf{x})\left(\nabla^{\prime} p_{p}^{2}+\nabla_{p}^{\prime \prime 0}\right) .
$$

Taking the average on $\Omega$ gives

$$
\left\langle\mathbf{v}_{p}^{2}\right\rangle_{\Omega}-n \mathbf{v}_{s}^{2}=-\mathbf{K}_{p}\left(\nabla^{\prime} p_{p}^{2}+\nabla_{p}^{\prime \prime 0}\right), \quad \mathbf{K}_{p}=\left\langle\mathbf{k}_{p}\right\rangle \Omega .
$$

- The mass balance (A.7d) $\nabla \mathbf{v}_{p}^{3}+\nabla^{\prime} \mathbf{v}_{p}^{2}+\nabla^{\prime \prime} \mathbf{v}_{p}^{0}=0$, gives by integration on $\Omega_{p}$ and (A.13d)

$$
n \nabla^{\prime \prime} \mathbf{v}_{p}^{0}+\nabla^{\prime}\left\langle\mathbf{v}_{p}^{2}\right\rangle_{\Omega}+\frac{1}{|\Omega|} \int_{\Gamma} \mathbf{v}_{s}^{3} \mathbf{N}_{p} \mathrm{~d} S=0
$$


Now we have

$$
\mathbf{v}_{s}^{3}=\frac{\partial \mathbf{u}_{s}^{3}}{\partial t}+\frac{\partial \mathbf{u}_{s}^{1}}{\partial T}
$$

Since $\mathbf{u}_{s}^{1}$ is $\mathbf{x}$-independent, the integral of $\partial \mathbf{u}_{s}^{1} / \partial T$ cancels out and we are left with

$$
\int_{\Gamma} \mathbf{v}_{s}^{3} \mathbf{N}_{p} \mathrm{~d} S=\int_{\Gamma} \frac{\partial \mathbf{u}_{s}^{3}}{\partial t} \mathbf{N}_{p} \mathrm{~d} S
$$

Introducing the expression (A.27) for $\mathbf{u}_{s}^{3}$, the fact that

$$
\mathbf{v}_{p}^{0}=\mathbf{v}_{s}^{0}\left(\mathbf{x}^{\prime \prime}, t, T\right)
$$

and

$$
\nabla^{\prime} \mathbf{v}_{s}^{2}=\nabla^{\prime}\left(\frac{\delta \mathbf{u}_{s}^{2}}{\delta t}+\frac{\delta \mathbf{u}_{s}^{0}}{\delta T}\right)=\nabla^{\prime} \frac{\delta \mathbf{u}_{s}^{2}}{\delta t},
$$

Equation (A.30) can be written after some calculations in the form

$$
\nabla^{\prime}\left(\left\langle\mathbf{v}_{p}^{2}\right\rangle_{\Omega}-n \mathbf{v}_{s}^{2}\right)=-\gamma^{\prime} \frac{\delta \mathbf{e}^{\prime \prime}\left(\mathbf{u}_{s}^{0}\right)}{\sigma t}-\beta \frac{\delta p^{0}}{\delta t} .
$$

- Finally, (A.11b) integrated on $\Omega_{f}^{\prime}$ gives

$$
\nabla^{\prime \prime}\left\langle\mathbf{v}_{f}^{0}\right\rangle_{\Omega^{\prime}}+\frac{1}{\left|\Omega^{\prime}\right|} \int_{\Gamma} \mathbf{v}_{f}^{2} \mathbf{N}^{\prime} \mathrm{d} S=0 .
$$

Using (A.16b) on $\Gamma^{\prime}$ the last integral becomes

$$
\begin{aligned}
\int_{\Gamma^{\prime}} \mathbf{v}_{f}^{2} \mathbf{N}_{f}^{\prime} \mathrm{d} S & =\int_{\Gamma^{\prime}}\left(\mathbf{v}_{s}^{2}+\left\langle\mathbf{v}_{p}^{2}\right\rangle\right) \mathbf{N}_{s p}^{\prime} \mathrm{d} S \\
& =-\int_{\Omega_{\mathrm{sp}}^{\prime}}\left(\nabla^{\prime} \mathbf{v}_{s}^{2}+\nabla^{\prime}\left\langle\mathbf{v}_{p}^{2}\right\rangle\right) \mathrm{d} \boldsymbol{\Omega}^{\prime} .
\end{aligned}
$$

And with the help of (A.31) we have

$$
\nabla^{\prime \prime}\left(\left\langle\mathbf{v}_{f}^{0}\right\rangle_{\Omega^{\prime}}-n^{\prime} \mathbf{v}_{s}^{0}\right)=-\gamma^{\prime \prime} \frac{\delta \mathbf{e}^{\prime \prime}\left(\mathbf{u}_{s}^{0}\right)}{\delta t}-\beta^{\prime \prime} \frac{\delta p^{0}}{\delta t}
$$

\section{References}

Auriault, J. L., 1991, Heterogeneous media. Is an equivalent macroscopic description possible? to appear in Int. J. Engng. Sci.

Auriault, J. L. and Sanchez-Palencia, E., 1977, Etude du comportement macroscopique d'un milieu poreux saturé déformable, J. Méchanique 16 (4), 575-603.

Barenblatt, G. I., Zheltov, Y. P., and Kochina, I. N., 1960, Basic concepts in the theory of seepage of homogeneous liquids in fissured rocks, P.M.M. 24(5), 852-864.

Biot, M. A., 1941, General theory of three-dimensional consolidation, J. Appl. Phys. 12, 155-164.

Levy, T., 1990, Filtration in a porous fissured rock: influence of the fissures connexity, Eur. J. Mech. B/Fluids 9, 309-327. 
Levy, T. and Sanchez-Palencia, E., 1975, On boundary conditions for fluid flow in porous media, Int. $J$. Engng. Sci. 13, 923-940.

Quintard, M. and Whitaker, S., 1987, Ecoulement monophasique en milieux poreux: effet des hétérogénités locales, J. Mécanique Théor. Appl. 6, 691-726.

Quintard, M. and Whitaker, S., 1988, Two-phase flow in heterogeneous porous media: the method of large scale averaging, Transport in Porous Media 3, 357-413.

Saez, A. E., Otero, C. J., and Rusinek, I., 1989, The effective homogeneous behavior of heterogeneous porous media, Transport in Porous Media 4, 213-238.

Van Golf-Racht, T. D., 1982, Fundamentals of Fractured Reservoir Engineering, Elsevier, New York.

Warren, J. E. and Root, P. J., 1963, The behaviour of naturally fractured reserovoirs, Soc. Petrol. Eng. $J ., 245-255$.

Wilson, R. K., and Aifantis, E. C., 1982, On the theory of consolidation with double porosity, Int. $J$. Engng. Sci. 20(9),1009-1035. 